\title{
Differences in the Peak Inspiratory Flow in Manual Breathing Assist and Rib Springing Techniques
}

\author{
Yoshihiro Yamashina $^{1}$, Tomoko Hirayama ${ }^{1}$, Hiroki Aoyama ${ }^{1}$, Hirofumi Hori ${ }^{1}$, Shota Fukunaga ${ }^{2}$, Masatoshi \\ Aso $^{2}$, Yukina Yamada ${ }^{2}$, Yuki Tsuruta ${ }^{3}$, Shiho Yamashina ${ }^{4}$, Emiko Morita ${ }^{1}$, Nami Sakagami ${ }^{1}$, Yosuke \\ Yamato $^{1}$, Hiroto Honda ${ }^{1}$, Shigeru Terada ${ }^{1}$, and Masahiro Goto ${ }^{1}$ \\ ${ }^{1}$ Department of Physical Therapy, Aino University (E-mail: y-yamashina@pt-u.aino.ac.jp) \\ ${ }^{2}$ Department of Physical Therapy, Azuma Medical Group \\ ${ }^{3}$ Department of Physical Therapy, Osaka Social Medical Center \\ ${ }^{4}$ Department of Physical Therapy, Long-Term Care Health Facilities Hureai
}

\begin{abstract}
Purpose: The present study aimed to compare the tidal volume and the peak inspiratory flow during manual breathing assist and rib springing in the lower chest. Method: Twenty-three healthy adult men were included. The manual breathing assist and rib springing procedures were performed by a physical therapist familiar with respiratory physical therapy. Measurement conditions were as follows: the maximum tidal volume was measured during quiet breathing, and while using manual breathing assist, and during rib springing. The peak inspiratory flow was measured 3 times each during manual breathing assist and rib springing, and the maximum value was used. Results: The tidal volume increased significantly during manual breathing assist and rib springing in comparison to quiet breathing. There was no significant difference in the tidal volume in manual breathing assist and rib springing. The peak inspiratory flow during rib springing was significantly higher compared to that during manual breathing assist. Conclusion: Our results suggest that the rib springing was able to increase the peak inspiratory flow by about 1.6 times in comparison to the manual breathing assist.
\end{abstract}

Keywords: manual breathing assist, rib springing, tidal volume, peak inspiratory flow, healthy adult males

\section{Introduction}

Respiratory assistance is a procedure performed by placing the palm on the patient's chest, and applying pressure in the direction of physiological motion during exhalation, and releasing the pressure during the following inhalation. The results of the procedure include increased tidal volume, improved ventilation, mitigated breathing difficulty, improved rib cage flexibility, and others ${ }^{1-6}$. It is one of the most popular and distinctive procedures for respiratory physical therapy in Japan ${ }^{5}$. On the other hand, rib springing is known as a distinct assistance method that accompanies basic respiratory assistance procedure, which is done by quickly releasing the hand in the direction of inspiratory movement of the chest at the early stage of inhalation after sufficient breathing has been induced. The procedure aims to induce deep breathing by utilizing the elasticity of the chest during expansion. It is considered effective in treating atelectasis, a complication disorder that often results from surgery on the respiratory tract, which involves the removal of obstructions due to bronchial foreign body or tumor, and sputum in the lungs and main bronchi due to rapid air inhalation. The inspiratory flow rate for the rib springing technique is thought to be higher than that of the basic respiratory aid procedure due to the rapid release of the pressure against the compressed chest. However, the extent of increase in the inspiratory flow rate is still unknown. Thus, this study is aimed to compare the tidal volume and peak inspiratory flow during respiratory assistance and rib springing in the lower chest.

Received: January 03, 2019

Accepted: May 14, 2019

Published: June 14, 2019 


\section{Materials and Methods}

\subsection{Approval from the Ethics Committee}

This study protocol was approved by the research ethics committee of Aino University (Aino2016 - 013).

\subsection{Subjects}

Twenty-three normal healthy adult males were included in the study. Subjects who had a history of respiratory or cardiovascular disease, hypertension [resting systolic blood pressure (BP) $\geq 140 \mathrm{mmHg}$ and/or diastolic $\mathrm{BP} \geq 90 \mathrm{~mm} \mathrm{Hg}$ ], diabetes, obesity [body mass index (BMI) $\geq 30 \mathrm{~kg} / \mathrm{m}^{2}$ ], or a habit of smoking were excluded. The eligible applicants who met the inclusion criteria participated in the study after familiarizing themselves with the experimental protocol, such as the measurement method using spirometry, as described below. The characteristics of the subjects are summarized in Table 1. Prior to entry into the study, written consent was obtained from all subjects after the provision of thorough written and oral explanations of the purpose, methods, and risks associated with the study.

Table 1 . The physical characteristics of the subjects

\begin{tabular}{lrc}
\hline Number & \multicolumn{2}{c}{23} \\
Age (years) & $25.6 \pm 2.7$ \\
Weight (kg) & 66.8 & \pm 6.2 \\
Height (cm) & 167.3 & \pm 3.9 \\
$\% \mathrm{VC}(\%)$ & 94.1 & \pm 6.2 \\
$\mathrm{FEV}_{1}(\%)$ & 90.7 & \pm 3.6 \\
\hline
\end{tabular}

Values are means $\pm \mathrm{SD}$.

Abbreviations: $\mathrm{VC}$, vital capacity; $\mathrm{FEV}_{1}$, forced expiratory volume in one second.

\subsection{Measurement of Tidal Volume and Peak Inspiratory Flow}

The manual breathing assist and rib springing procedures were performed by a physical therapist who was familiar with respiratory physical therapy (17 years of experience). The tidal volume was measured with an electronic spirometer AS-307, manufactured by MINATO Co., and the peak inspiratory flow rate in the supine position was measured using In-Check, manufactured by Clement Clarke Co. Measurement conditions were as follows: the maximum tidal volume was measured during quiet breathing, and while performing manual breathing assist, and rib springing techniques. The peak inspiratory flow rate was measured during manual breathing assist and rib springing for 3 times each, and the maximum value was used.

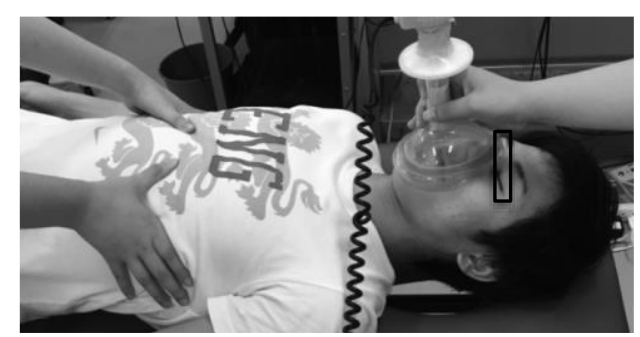

Fig1. Measurement of Tidal Volume

\subsection{Statistical Analysis}

For statistical analysis, the tidal volume was analyzed by one-way ANOVA followed by a multiple comparison test. The peak inspiratory flow rate was compared using a corresponding t-test.

A significance level of $<5 \%$ was used for all evaluations, and the analysis was performed with the StatView 
5.0 software for Windows (SAS Institute, Cary, NC, USA).

\section{Results}

The tidal volume increased significantly during manual breathing assist and rib springing compared to quiet breathing. There was no significant difference in the tidal volume during manual breathing assist and rib springing. The peak inspiratory flow in rib springing was significantly higher compared to that in manual breathing assist.

Table 2.Change of TV and PIF.

\begin{tabular}{|c|c|c|c|c|}
\hline & quite breathing & manual breathing assist & \multicolumn{2}{|c|}{ rib springing } \\
\hline TV (L) & $0.74 \pm 0.34$ & $1.04 \pm 0.38^{*}$ & $1.13 \pm$ & $0.40^{*}$ \\
\hline PIF (L/min) & & $43.7 \pm 16.2$ & $68.2 \pm$ & $21.1^{\#}$ \\
\hline
\end{tabular}

Values are means $\pm \mathrm{SD} .{ }^{*} \mathrm{p}<0.05$ vs. quite breathing. ${ }^{*} \mathrm{p}<0.05$ vs. manual breathing assist.

Abbreviations: TV, tidal volume; PIF, peak inspiratory flow.

\section{Discussion}

This study has reconfirmed that manual breathing assist and rib springing techniques are effective in increasing ventilation air volume, and has clarified that the rib springing technique can significantly increase the peak inspiratory flow compared to manual breathing assist.

Generally, respiratory assistance procedures, including rib springing technique, have been shown to be effective in improving ventilation ${ }^{1-6}$. The results obtained in our study further confirms that both techniques are effective in improving ventilation volume, in line with previous studies, by inducing deep inhalation through the elastic force of the chest, which is generated by the assisting chest wall movement during exhalation and alleviating the assistance during inhalation. We think that the implementation of these procedures in clinical situations, where a patient's voluntary respiratory effort is not sufficient or possible, will be able to promote gas exchange, improve ventilation by moving the accumulated airway secretions, and prevent or treat atelectasis.

We showed that the peak inspiratory flow rate during rib springing is about 1.6 times of that during manual breathing assist. The major difference between rib springing and manual breathing assist is in the method to alleviate assistance during inhalation. Manual breathing assist slowly relieves the assistance, whereas rib springing relieves assistance swiftly. Thus, it may be inferred that the elastic force of the chest works better in rib springing than in manual breathing assist, causing the inspiratory flow rate to be higher in rib springing. This rapid inhalation should better assist the movement of sputum in the airway, suggesting that the technique is useful for atelectasis treatment.

However, in rib springing, since the hands are quickly removed in the direction of inspiratory movement of the chest early in inhalation after sufficient breathing is induced, the procedure may cause pain on the chest or damage to the lung and the bronchi due to the high pressure applied to the chest. Therefore, when an inexperienced medical worker performs rib springing, the timing of the patient's respiration may not match with that of the respiration assistance procedure, which may cause breathing disturbances and discomfort to the patient. Furthermore, since there seems to be a relatively high risk of inducing arrhythmia and rib fracture due to rib springing, it is necessary to provide sufficient training to master the skills.

\subsection{Limitations}

There are some limitations of the present study, which includes its relatively small cohort comprised of only healthy male subjects.

It is thought that the thoracic flexibility of the subjects affects the peak inspiratory flow ${ }^{7), 8}$, though we did not evaluate the same this time. Therefore, a further study is necessary to clarify the effect of thoracic flexibility on the peak inspiratory flow.

We have not conducted research combining manual breathing assist and rib springing in this study. Therefore, the combined effects of these techniques are not clarified. However, We have to examine the effect of the combination of respiratory assistance and rib springing in the future since these techniques are often 
used in conjunction for the patients clinically.

\section{Conclusion}

We examined the differences in the ventilation volume and the peak inspiratory flow during manual breathing assist and rib springing procedure. Although both procedures could increase the ventilation volume, the rib springing technique was able to increase the peak inspiratory flow by about 1.6 times higher than the manual breathing assist.

\section{Acknowledgements}

We are grateful to members of Aino University for their important contributions to this experiment. We would like to thank Editage (www.editage.jp) for English language editing.

\section{Conflicts of Interest Statement}

The authors declare no conflicts of interest in relation to this article.

\section{References}

1) Kurita, H., Nitta, O., Harada, M., \& Mizukami, M. 2010. Ventilatory effects of manual breathing assist technique (MBAT) and shaking in central nervous system disease sufferers. J. Phys. Ther. Sci., 22: 209-15.

2) Ihashi, K., Saitou, A., Yahata, J., \& Ito, N. 1989. Effect of manual breathing assist technique on lung volumes. J. Phys. Ther.,16: 267-72.

3) Kihara, K., Mase, K., Nozoe, M., Okada, M., Murakami, S., Ogino, T., Matsushita, K., Kajisa, N., Wada, T., Mabuchi, S., Terayama, S., Hukuda, Y., \& Doumen, K. 2015. Features of palm pressure distribution during the breathing assist technique. J. Jap. Soci. Res. Reha., 25: 309-14.

4) Kolaczkowski, W., Taylor, R., \& Hoffstein, V. 1989. Improvement in oxygen saturation after chest physiotherapy in patients with emphysema. Physi. Can., 41: 18-23.

5) Tanaka, T., Kozu, R., Kitagawa, C., Asai, M., \& Senjyu, H. 2015. A manual breathing assist proficiency scale for therapists interrater reliability and elements which is related to tidal volume. J. Jap. Soci. Res. Reha., 25: 95-98.

6) McCarren, B., Alison, JA.,\& Herbert, RD. 2006. Manual vibration increases expiratory flow rate via increased intrapleural pressure in healthy adults: an experimental study. Aust. J. Physiother., 52: 267-71.

7) Leelarungrayub, D., Pothongsunun, P., Yankai, A., \& Pratanaphon, S. 2009. Acute clinical benefits of chest wallstretching exercise on expired tidal volume, dyspnea and chest expansion in a patient with chronic obstructive pulmonary disease: a single case study. J. Bodyw. Mov. Ther., 13: 338-43.

8) Rattes, C., Campos, S.L., Morais, C., Gonçalves, T., Sayão, L.B., Galindo-Filho, V.C., Parreira, V., Aliverti, A., \& Dornelas de Andrade, A. 2018. Respiratory muscles stretching acutely increases expansion in hemiparetic chest wall. Respir. Physiol. Neurobiol., 254: 16-22. 